LIMA, L.L.; NUNES; G.H.S.; BEZERRA NETO, F. Coeficientes de variação de algumas características do meloeiro: uma proposta de classificação. Horticultura Brasileira, Brasília, v.22, n.1, p.14-17, jan-mar 2004.

\title{
Coeficientes de variação de algumas características do meloeiro: uma proposta de classificação
}

\author{
Lonjoré Leocádio de Lima; Glauber Henrique de S. Nunes; Francisco Bezerra Neto \\ ESAM, C. Postal 137, 59625-900 Mossoró-RN; bezerra@esam.br
}

\section{RESUMO}

Uma proposta para classificação dos coeficientes de variação das características: produtividade, peso do fruto, número de frutos, teor de sólidos solúveis, firmeza da polpa, açúcares totais e acidez total titulável foi estabelecida em dados de trabalhos com meloeiro (Cucumis melo L.) conduzidos na cidade de Mossoró. Os valores de CVs foram obtidos de 98 trabalhos, em sua maioria dissertações e monografias defendidas na ESAM. Foram utilizados os métodos de Garcia (1989) e dos Quantis Amostrais. Houve discordância nas freqüências observadas entre as duas metodologias propostas neste trabalho e a de Pimentel Gomes (1985) para as características açúcar total, teor de sólidos solúveis, peso do fruto, produtividade e número de frutos. Para a acidez total titulável, observaram-se reduzidas diferenças quanto aos limites baixo, médio e alto, porém, segundo a classificação de Pimentel Gomes (1985), nenhum dos dados foi classificado como muito alto. Apenas para a característica firmeza da polpa houve alta concordância entre as metodologias de Garcia (1985) e dos Quantis Amostrais e a de Pimentel Gomes (1985). Os métodos de Garcia (1985) e dos Quantis Amostrais permitiram a classificação mais adequada dos coeficientes de variação para o meloeiro.

Palavras-chave: Cucumis melo, precisão experimental, análise de variância.

\begin{abstract}
Coefficients of variation of some melon yield components and fruit quality traits: a proposal for classification

A proposal for classifying the coefficients of variation of melon yield, fruit weight, fruit number, soluble solids content, pulp firmness, total sugars, $\mathrm{pH}$ and total titrable acidity was established in data from melon experiments carried out in Mossoró, Brazil. The CV values came from 98 works, mostly from theses and monographs submitted to the faculty of Mossoró. The variation coefficients (CV) were classified according to Garcia's (1989) proposal and sample quantiles. A disagreement in the observed frequencies between these two methodologies proposed in this paper and that of Pimentel Gomes (1985) for total sugars, soluble solids content, fruit weight, $\mathrm{pH}$, yield and number of fruits was observed. Small differences were observed in the low, medium and high $\mathrm{CV}$ bands for total titrable acidity. However, very high $\mathrm{CV}$ band for any assessed characteristic, based on the Pimentel Gomes (1985) classification was not observed. A very good agreement between the classifications proposed by Garcia (1989) and sample quantiles, and that of Pimentel Gomes (1985) for pulp firmness was observed. In general, the classifications proposed by Garcia (1989) and sample quantiles were more appropriate to evaluate the melon yield and quality traits than that of Pimentel Gomes (1985).
\end{abstract}

Keywords: Cucumis melo, experimental precision, analysis of variance.

(Recebido para publicação em 17 de dezembro de 2002 e aceito em 10 de novembro de 2003)

$\mathrm{E}_{\mathrm{c}}^{\mathrm{m}}$ m experimentação agrícola a preocupação com a precisão dos ensaios é constante por parte dos pesquisadores. Os ensaios com baixa precisão podem fazer com que se obtenham conclusões incorretas dos resultados, aumentando-se a probabilidade de ocorrência de erro tipo II, ou seja, apontar igualdade entre tratamentos quando realmente existe diferença. Com efeito, a decisão errada prejudica a adoção de novas tecnologias por não permitir a indicação do melhor tratamento para o produtor (Judice, 2000).

O coeficiente de variação $(\mathrm{CV})$, definido como o desvio-padrão expresso em porcentagem de média, é a medida mais utilizada para medir a instabilidade relativa de uma característica ou variável (Sampaio, 1998). Considera-se que quanto menor o $\mathrm{CV}$, maior será a homogeneidade dos dados e menor a variação do acaso (Garcia, 1989).

$\mathrm{Na}$ experimentação agrícola, o CV tem a vantagem de possibilitar a comparação da precisão entre experimentos, envolvendo a mesma variável-resposta, permitindo que o pesquisador possa quantificar a precisão de sua investigação (Steel e Torrie, 1980; Sampaio, 1998). Contudo, deve-se tomar cuidado, com resultados de experimentos distintos que são obtidos em condições diferentes (Scapim et al., 1995).

Pimentel Gomes (1985) fez uma classificação para o coeficiente de variação. No entanto, essa classificação, mesmo sendo extensivamente utilizada, é abrangente e não considera a natureza do ensaio quanto à cultura estudada $\mathrm{e}$, principalmente, quanto à variável utilizada, o que pode ser importante para interpre- tação das magnitudes dessa medida, conforme comentou Garcia (1989).

Existe a necessidade de classificação dos coeficientes de variação para a realidade inerente a cada cultura, a fim de que se possa ter um referencial na comparação da precisão de experimentos distintos. Alguns trabalhos neste sentido foram realizados com milho (Scapim et al., 1995), citros (Amaral et al., 1997), soja e trigo (Lúcio, 1997), arroz (Costa et al., 2002), espécies forrageiras (Ambrosano e Schamas, 1994; Clemente e Muniz, 2002), espécies florestais (Garcia, 1995) e diversas culturas agrícolas (Estefanel et al., 1987). Na área animal, também há trabalhos que visam a classificação de coeficientes de variação (Judice et al., 1999).

Para melão, uma das principais hortaliças cultivadas no Nordeste brasileiro, não existe uma classificação especí- 
Tabela 1. Teste de Shapiro-Wilk (SW) e estatísticas descritivas dos coeficientes de variação obtidos em trabalhos com a cultura do melão (Cucumis melo L.). Mossoró, 2001.

\begin{tabular}{lccccccccc}
\hline Variável & $\mathbf{n}^{\mathbf{1}}$ & sw & Média & $\begin{array}{c}\text { Desvio } \\
\text { padrão }\end{array}$ & Max & Min & Assimetria & Curtose & CV(\%) \\
\hline AT & 52 & $0,49^{\text {ns2 }}$ & 11,61 & 5,76 & 25,58 & 1,46 & $0,13 \mathrm{~ns}$ & $-0,59 \mathrm{~ns}$ & 46,83 \\
FP & 62 & $0,65 \mathrm{~ns}$ & 14,96 & 5,66 & 31,70 & 3,92 & $0,72^{*}$ & $0,61 \mathrm{~ns}$ & 37,96 \\
TSS & 99 & $0,27 \mathrm{~ns}$ & 11,96 & 3,49 & 18,20 & 4,61 & $-0,16 \mathrm{~ns}$ & $-0,52 \mathrm{~ns}$ & 29,18 \\
PROD & 53 & $0,10 \mathrm{~ns}$ & 28,19 & 14,79 & 51,30 & 4,44 & $0,45 \mathrm{~ns}$ & $0,64 \mathrm{~ns}$ & 52,47 \\
PF & 47 & $0,32 \mathrm{~ns}$ & 10,66 & 2,87 & 18,40 & 6,03 & $0,35 \mathrm{~ns}$ & $-0,09 \mathrm{~ns}$ & 25,58 \\
NF & 42 & $0,56 \mathrm{~ns}$ & 19,21 & 7,27 & 34,13 & 4,13 & $0,25 \mathrm{~ns}$ & $-0,49 \mathrm{~ns}$ & 37,84 \\
ATT & 52 & $0,34 \mathrm{~ns}$ & 13,34 & 4,40 & 22,14 & 4,58 & $0,11 \mathrm{~ns}$ & $-0,57 \mathrm{~ns}$ & 32,95 \\
\hline
\end{tabular}

AT: Açucares totais; FP: Firmeza da polpa; TSS: Teor de sólidos solúveis; PROD: Produtividade; Peso do fruto; Número de frutos; ATT: Acidez total titulável

1 Tamanho da amostra

2 Não significativo a $5 \%$ de probabilidade

fica. Assim sendo, este trabalho teve como objetivo classificar os coeficientes de variação de experimentos com essa cultura.

\section{MATERIAL E MÉTODOS}

Os valores de CVs foram obtidos de 98 trabalhos com a cultura do meloeiro, em sua maioria, em teses e monografias defendidas na ESAM, além de periódicos nacionais e internacionais. As características avaliadas foram aquelas relacionadas à produção (produtividade, peso do fruto e número de frutos) e à qualidade de frutos (teor de sólidos solúveis, firmeza da polpa, açúcar total e acidez total titulável). Inicialmente, testou-se o ajuste dos dados à distribuição normal pelo teste de Shapiro-Wilk (Shapiro e Wilk, 1965). Foram obtidas estimativas de medidas descritivas como média geral, desvio-padrão, valores máximos e mínimos, coeficientes de assimetria e curtose, e coeficiente de variação. A classificação foi feita de acordo com as propostas de Garcia (1989) e dos Quantis Amostrais.

A metodologia de Garcia (1989) utiliza a relação entre a média $(\mathrm{m})$ e o desvio padrão (DP) dos valores do $\mathrm{CV}$ de diversos experimentos, envolvendo a mesma variável, classificando-os da seguinte maneira: baixo $[(\mathrm{CV} \leq(\mathrm{m}-1 \mathrm{DP})]$; médio $[(\mathrm{m}-1 \mathrm{DP})<\mathrm{CV}<(\mathrm{m}+1 \mathrm{DP})]$; alto $[(\mathrm{m}+1 \mathrm{DP})<\mathrm{CV} \leq(\mathrm{m}+2 \mathrm{DP})] \mathrm{e}$ muito alto $[\mathrm{CV}>(\mathrm{m}+2 \mathrm{DP})]$.

No método dos Quantis Amostrais os dados foram organizados em ordem cres- cente de grandeza e encontrados os valores $15,87 \% ; 84,13 \%$ e $97,72 \%$ que, analogamente ao critério de Garcia (1989), foram utilizados como estimativas das faixas de avaliação dos CVs dos experimentos (Spiegel, 1996; Judice 2000).

Foram construídas tabelas de freqüências dos CVs segundo a classificação proposta pelos métodos de Garcia (1989), dos Quantis Amostrais e pela classificação de Pimentel Gomes (1985), a partir dos dados obtidos na literatura consultada.

\section{RESULTADOS E DISCUSSÃO}

Os resultados para as estatísticas descritivas e teste de normalidade de Shapiro- Wilk estão apresentados na Tabela 1. Observou-se que o teste de Shapiro-Wilk não foi significativo para as características estudadas, evidenciando que o conjunto de dados dos coeficientes de variação extraídos da literatura tiveram distribuição normal. $\mathrm{O}$ fato das estimativas dos coeficientes de assimetria e curtose não serem significativos, indica que a distribuição dos dados é simétrica e mesocúrtica, características da curva de Gauss (Triola, 1998).

De um modo geral, constata-se uma tendência das características medidas em laboratório (SST, ATT, Açucares e Firmeza) terem menores CVs em relação àquelas determinadas em campo (Produtividade e Número de frutos). Entretanto, o peso do fruto foi o segundo menor coeficiente de variação (Tabela 1). Amaral et al. (1997), trabalhan- do com citros, também observaram que as características medidas em laboratório, com exceção da acidez, possuíam maiores estimativas de $\mathrm{CV}$ em relação às de campo. Segundo esses autores, as características determinadas em laboratório têm menores valores em decorrência, talvez, da maior precisão com que este tipo de análise é conduzida, como maior controle dos fatores ambientais e o uso de instrumentos precisos.

Ocorreu ampla variação entre os CVs dentro de cada característica, nos valores máximo e mínimo e nos coeficientes de variação dos dados. A variação dos coeficientes de variação entre experimentos é função das condições distintas nas quais foram realizados os experimentos. Em razão dessa variabilidade, é necessária a classificação específica dos CVs de cada característica.

Assim sendo, para cada variável foi elaborada uma faixa específica de valores de coeficientes de variação específica, conforme os critérios de Garcia (1989) e dos Quantis Amostrais (Tabela 2). Observou-se que os resultados obtidos são bastante semelhantes nos dois métodos, que foram recomendados por Judice (2000) para classificação de CVs em experimentos zootécnicos porque consideram a real distribuição dos dados.

As freqüências observadas e esperadas, segundo as metodologias de Garcia (1989), dos Quantis Amostrais e a classificação proposta por Pimentel Gomes (1985), estão na Tabela 3. As freqüências esperadas foram estimadas considerando-se que os coeficien- 
Tabela 2. Intervalos dos coeficientes de variação construídos pelos critérios de Garcia (G) (1989) e dos Quantis Amostrais (QA) a partir dos dados obtidos em trabalhos com a cultura do melão (Cucumis melo L.). Mossoró, 2001.

\begin{tabular}{|c|c|c|c|c|c|}
\hline \multirow{2}{*}{ Critério } & \multirow{2}{*}{ Característica } & \multicolumn{4}{|c|}{ Classificação do CV } \\
\hline & & ( $\leq$ ) Baixo & Médio & Alto & Muito Alto (>) \\
\hline \multirow{7}{*}{ G } & AT & 5,85 & $5,85<\mathrm{CV} \leq 19,37$ & $19,37<\mathrm{CV} \leq 23,85$ & 23,85 \\
\hline & FP & 9,30 & $9,30<\mathrm{CV} \leq 20,20$ & $20,20<C V \leq 29,12$ & 29,12 \\
\hline & SST & 7,59 & $7,59<\mathrm{CV} \leq 14,90$ & $14,90<\mathrm{CV} \leq 17,28$ & 17,28 \\
\hline & PROD & 13,45 & $13,45<\mathrm{CV} \leq 45,79$ & $45,79<\mathrm{CV} \leq 51,30$ & 51,30 \\
\hline & PF & 7,79 & $7,79<\mathrm{CV} \leq 13,97$ & $13,97<\mathrm{CV} \leq 17,71$ & 17,71 \\
\hline & NF & 12,25 & $12,25<\mathrm{CV} \leq 26,77$ & $26,77<\mathrm{CV} \leq 33,18$ & 33,18 \\
\hline & ATT & 8,07 & $8,07<\mathrm{CV} \leq 17,71$ & $17,71<\mathrm{CV} \leq 21,11$ & 21,17 \\
\hline \multirow{7}{*}{ QA } & AT & 6,73 & $6,73<\mathrm{CV} \leq 18,06$ & $18,06<\mathrm{CV} \leq 23,82$ & 23,82 \\
\hline & FP & 9,25 & $9,25<C V \leq 20,57$ & $20,57<C V \leq 26,23$ & 26,23 \\
\hline & SST & 8,47 & $8,47<\mathrm{CV} \leq 15,45$ & $15,45<C V \leq 18,94$ & 18,94 \\
\hline & PROD & 13,4 & $13,4<\mathrm{CV} \leq 42,98$ & $42,98<\mathrm{CV} \leq 57,77$ & 57,77 \\
\hline & $\mathrm{PF}$ & 7,79 & $7,79<C V \leq 13,97$ & $13,97<\mathrm{CV} \leq 17,71$ & 17,71 \\
\hline & NF & 11,94 & $11,94<\mathrm{CV} \leq 26,48$ & $26,48<\mathrm{CV} \leq 33,75$ & 33,75 \\
\hline & ATT & 8,94 & $8,94<\mathrm{CV} \leq 17,72$ & $17,72<\mathrm{CV} \leq 22,11$ & 21,11 \\
\hline
\end{tabular}

AT: Açucares totais; FP: Firmeza da polpa; TSS: Teor de sólidos solúveis; PROD: Produtividade; Peso do fruto; Número de frutos; ATT: Acidez total titulável

Tabela 3. Freqüências observadas e esperadas (\%) dos coeficientes de variação segundo os critérios de Garcia (1989) (G), dos Quantis Amostrais (QA) e Pimentel Gomes (1985) (PG) a partir dos dados obtidos em trabalhos com a cultura do melão (Cucumis melo L.). Mossoró, 2001.

\begin{tabular}{|c|c|c|c|c|c|c|c|c|c|}
\hline \multirow{2}{*}{ Critério } & \multirow{2}{*}{ Classificação } & \multirow{2}{*}{$F_{e}^{1}(\%)$} & \multicolumn{7}{|c|}{$F_{0}(\%)^{2}$} \\
\hline & & & $\overline{\text { AT }}$ & FP & TSS & PROD & PF & NF & ATT \\
\hline \multirow{4}{*}{ G } & Baixo & 15,86 & 17,31 & 12,90 & 16,16 & 13,21 & 21,0 & 12,00 & 21,00 \\
\hline & Médio & 68,27 & 65,38 & 72,58 & 67,68 & 68,00 & 68,00 & 71,00 & 65,00 \\
\hline & Alto & 13,59 & 13,46 & 12,90 & 16,00 & 15,00 & 6,00 & 14,29 & 23,08 \\
\hline & Muito Alto & 2,28 & 3,85 & 1,61 & 1,16 & 3,00 & 4,00 & 2,71 & 1,92 \\
\hline \multirow{4}{*}{ QA } & Baixo & 15,86 & 15,38 & 16,13 & 15,15 & 15,09 & 14,89 & 16,67 & 15,38 \\
\hline & Médio & 68,27 & 69,23 & 67,74 & 67,67 & 68,81 & 70,21 & 66,67 & 69,23 \\
\hline & Alto & 13,59 & 13,46 & 14,52 & 14,14 & 13,21 & 12,77 & 14,29 & 13,46 \\
\hline & Muito Alto & 2,28 & 1,92 & 1,61 & 3,03 & 1,88 & 2,13 & 2,38 & 1,92 \\
\hline \multirow{4}{*}{ PG } & Baixo & 15,86 & 40,38 & 36,73 & 36,73 & 11,32 & 31,91 & 9,90 & 19,61 \\
\hline & Médio & 68,27 & 53,85 & 61,67 & 61,22 & 24,53 & 68,09 & 45,46 & 68,63 \\
\hline & Alto & 13,59 & 7,70 & 16,67 & 0 & 22,64 & 0 & 29,55 & 13,73 \\
\hline & Muito Alto & 2,28 & 0 & 1,67 & 0 & 0,40 & 0 & 11,36 & 0 \\
\hline
\end{tabular}

AT: Açucares totais; FP: Firmeza da polpa; TSS: Teor de sólidos solúveis; PROD: Produtividade; Peso do fruto; Número de frutos; ATT: Acidez total titulável.

1 Freqüência esperada

2 Freqüência observada

tes de variação obtidos na experimentação com melão tenham distribuição normal ou aproximadamente normal. Portanto, espera-se que, sob a hipótese de normalidade, a maioria dos coeficientes de variação estejam próximos da média e que coeficientes de variação muito baixos ou muito elevados sejam pouco freqüentes.

Houve concordância para as freqüências observadas nos critérios de Garcia (1989) e de Quantis Amostrais, em todas as variáveis. Por outro lado, verificou-se discordância entre as duas metodologias propostas neste trabalho e aquela proposta por Pimentel Gomes (1985) para as características açúcares totais, teor de sólidos solúveis e peso do fruto. 
Para o teor de sólidos solúveis e peso do fruto, conforme Pimentel Gomes (1985), nenhum dos dados seria classificado como alto ou muito alto (Tabela 3), enquanto que, para a acidez total não houve CV classificado como muito alto.

Com relação à produtividade e número de frutos observaram-se diferenças quanto aos limites médio alto e muito alto (Tabela 3). Para produtividade, não houve $\mathrm{CV}$ classificado como muito alto, conforme Pimentel Gomes (1985).

Para a característica acidez total titulável, constataram-se reduzidas diferenças quanto aos limites baixo, médio e alto para os três métodos. Contudo, segundo a classificação de Pimentel Gomes (1985), nenhum dos dados seria classificado como muito alto.

Apenas para a característica firmeza da polpa, houve alta concordância entre as metodologias de Garcia (1985) e dos Quantis Amostrais e a de Pimentel Gomes (1985).

Trabalhando com milho, Scapim et al. (1995) verificaram concordância entre as metodologias de Garcia (1989) e de Pimentel Gomes (1985) para peso de espigas, número de espigas, peso de grãos e prolificidade. Com efeito, a classificação de Pimentel Gomes (1985) pode ser adequada para determinadas características.
A aplicação dos métodos de Garcia (1985) e dos Quantis Amostrais permitiu a classificação mais adequada dos coeficientes de variação para o melão, confirmando a necessidade de uma abordagem específica dessa medida de variação, em função da natureza dos dados, isto é, da característica em estudo, e da própria cultura. Esse fato foi verificado em outros trabalhos (Scapim et al., 1985; Amaral et al., 1997; Judice, 2000).

\section{LITERATURA CITADA}

AMARAL, A.M.; MUNIZ, J.A.; SOUZA, M. Avaliação do coeficiente de variação como medida da precisão na experimentação com citros. Pesquisa Agropecuária Brasileira, Brasília, v.32, p.1221-1225, dez. 1997.

AMBROSANO, G.M.B.; SCHAMMAS, E.A. Avaliação dos coeficientes de variação em experimentos com forrageiras. Boletim da Indústria Animal, São Paulo, v.51, n.1, p.13-20, 1994.

COSTA, N.H.A.D.; SERAPHIN, J.C.; ZIMMERMANN, F.J.P. Novo método de classificação de coeficientes de variação para a cultura do arroz de terras altas. Pesquisa Agropecuária Brasileira, Brasília, v.37, n.3, p.243-249, 2002.

CLEMENTE, A.L.; MUNIZ, J.A. Avaliação do coeficiente de variação em experimentos com gramíneas forrageiras. Ciência e Agrotecnologia, Lavras, v.26, n.1, p.197-203, 2002.

ESTEFANEL, V.; PIGNARATO, I.A.B.; STORCK, L. Avaliação do coeficiente de variação de experimentos com algumas culturas agrícolas. In: SIMPÓSIO DE ESTATÍSTICA APLICADA À EXPERIMENTAÇÃO AGRONÔMICA, 2., 1987, Londrina. Anais ... Londrina: FUEL/ RBRAS/IAPAR, 1987. p.115-131.
GARCIA, C.H. Tabelas para classificação de coeficientes de variação. Piracicaba: IPEF, 1989. 12 p. (Circular Técnica, 171).

JUDICE, M.G.; MUNIZ, J.A.; CARVALHEIRO, R. Avaliação do coeficiente de variação na experimentação com suínos. Ciência e Agrotecnologia, Lavras, v.23, n.1, p.170-173, 1999.

JUDICE, M.G. Avaliação de coeficiente de variação em experimentos zootécnicos. Lavras: UFLA, 2000. 40 p. (Tese mestrado)

LÚCIO, A.D. Parâmetros da precisão experimental das principais culturas anuais do Estado do Rio Grande do Sul. Santa Maria: UFSM, 1997. 62 p. (Tese mestrado)

PIMENTEL GOMES, F. Curso de Estatística Experimental. São Paulo: Nobel, 1985. 467 p.

SAMPAIO, I.B.M. Estatística aplicada à experimentação animal. Belo Horizonte: Fundação de Ensino e Pesquisa em Medicina Veterinária e Zootecnia, 1998. $221 \mathrm{p}$.

SCAPIM, C.A. ; CARVALHO, C.G.P.; CRUZ, C.D. Uma proposta de classificação dos coeficientes de variação para a cultura do milho. Pesquisa Agropecuária Brasileira, Brasília, v.30, p.683686, 1995.

SHAPIRO, S.S.; WILK, M.B. An analysis of variance test of normality (complete samples). Biometrika, v.52, n.3-4, p.591-611, 1965.

SPIEGEL, M.R. Estatística. Trad. e Rev. Técnica Pedro Consentino. 3 ed. São Paulo: Makron Books, 1996. 643 p. (Coleção Schaum).

STEEL, R.G.D.; TORRIE, J.H. Principles and procedures of statistics: with reference to the biological sciences. New York: Mc Graw-Hill, $1980.633 \mathrm{p}$

TRIOLA, M.F. Introdução à Estatística. Trad. Alfredo Alves de Faria e Ver. Técnica Eliana Farias e Soares. 7 ed. Rio de Janeiro: Livros técnicos e Científicos. 1998. 410 p. 